\title{
Pharmacovigilance studies on the basic knowledge, practice and attitude among the second year MBBS students of Jawaharlal Nehru institute of medical sciences, Porompat, Manipur, India
}

\author{
Varkung Valte, Babycha Laishram, Premchand M. Singh*, Indira Raleng
}

\begin{abstract}
Department of Pharmacology, Jawaharlal Nehru Institute of Medical Sciences, Imphal, Manipur, India
\end{abstract}

\section{Received: 01 March 2016 \\ Revised: 15 April 2016 \\ Accepted: 10 May 2016 \\ *Correspondence to: \\ Dr. Premchand M. Singh, Email: drpremcs@gmail.com \\ Copyright: (C) the author(s), publisher and licensee Medip Academy. This is an open- access article distributed under the terms of the Creative Commons Attribution Non- Commercial License, which permits unrestricted non- commercial use, distribution, and reproduction in any medium, provided the original work is properly cited.}

\begin{abstract}
Background: The recent advancement in the field of Medicine and Pharmaceutical sciences, the adverse effects of Medicine is a challenge among the medical fraternity. The objective of this study was to study the knowledge, Practice and attitude of ADRs among the second year MBBS students with the aim to find out the means for implementation of pharmacovigilance programme of India (PvPi).

Methods: The pretested questionnaire on knowledge, practice, attitude on Pharmacovigilance. The design of the study is cross sectional study. Percentage, proportions and means are used for descriptive statistics while the associations are calculated using corresponding tests for the associations.

Results: The knowledge of the students on pharmacovigilance in connection with over the counter/self-medication(52\%); minimum need of surveillance on marketing(74\%); present surveillance on marketing $60 \%$; need of CME on ADRs along with pharmacovigilance among student $(64 \%)$ as the ADRs on elderly (57\%), children (58\%), Pregnancy (64\%). Out of 24 questions on attitude, only 7 questions on reporting, multinational company, drug controller of India, disability and compensation are selected for statistical analysis. The percentage of the students who has heard and seen ADRs is 64.5.

Conclusions: If there is good communication and reports; most of the ADRs are avoidable and plays a pivotal role in minimising the ADRs. Drugs must be prescribed rationally and poly pharmacy should be discouraged and avoided as much as possible. To avoid the ADRs, pharmacovigilance is a matter of great concern for the health care providers and for the general mass too.
\end{abstract}

Keywords: PvPi, ADRs, UMC-WHO, CME, OTC, DC- I, MNC

\section{INTRODUCTION}

ADR is an increasing worldwide problem causing significant morbidity and mortality. Even with the administration of right dose of the right medicine through the right route at the right time of the rightly diagnosed patient, the patient may have side effects and adverse drug reactions. As per the WHO, ADR is a response of a drug which is noxious/ unintended and which occurs at doses normally used in man for the prophylaxis, diagnosis or therapy of disease and for the modification of physiological functions. ${ }^{1}$ Therefore, monitoring of ADRs has become exceedingly important in today's practice of medicine particularly in a developing country like India. On the other hand, Pharmacovigilance is the activities relating to the detection, assessment, understanding and prevention of ADRs or any other drug related problems. ${ }^{1}$ It is not enough to measure the incidence of adverse reactions to drugs, their nature and their severity, though accurate data are obviously useful. It is necessary to take, or to try to take, into account which effects are avoidable (by skilled choice and use) and which are unavoidable (inherent in drug or patient). Also, different adverse effects can matter to a different degree to different people. ${ }^{2}$

When unexpected effects are seen, a drug interaction should be suspected. Careful drug histories are important; 
because patients may take over-the-counter drugs or herbal products, take drugs prescribed by another physician, or take drugs prescribed for another patient. Care must be exercised when major changes are made in a drug regimen, and drugs that are not necessary should be discontinued. When an interaction is discovered, the interacting drugs often may be used effectively with adjustment of dosage or other therapeutic modifications. ${ }^{3}$

Drugs are, or should be, prescribed or taken with the object of alleviating symptoms or other manifestations of ill health. It is therefore legitimate for some risk to be incurred when drugs are given, provided that at the same some benefit is likely to be conferred upon the recipient. ${ }^{4}$

Drug toxicity or adverse reactions to drugs may be defined as any response to a drug that is noxious and unintended and which occurs at doses used in humans for prophylaxis, diagnosis and therapy. ${ }^{5}$

Data collection, data processing and analysis are the pillars of Pharmacovigilance. The primary source of the ADRs is the spontaneous reporting of the health care professionals and even by the patients. Sometimes the drug may be withdrawn during the clinical trial and from the post marketing surveillance. The primary object of statistical analysis in pharmacological studies is to find out whether the effect produced by a compound under study is genuine and not due to chance. ${ }^{6}$

WHO-UMC the Uppsala monitoring centre Sweden is the international pharmacovigilance centre where the international database of adverse drug reactions is maintained. In India, the pharmacovigilance (PvPi) programme is started with the aims of generating ADRs database. $^{7}$ On the basis of ADRs/pharmacovigilance report many drugs were withdrawn from the market. Initiative and willingness of health care providers through voluntary reporting, after prescription event monitoring, computerised medical record linkage; dissemination of ADR data (drug alerts, medical letters) with changing in labelling, statuary warning and precaution will prevent many ADRs. PvPi is a matter of great concern for the health care providers and the general mass too. It has an important role in the rational use of drugs as it provides the basis for assessing the safety of drugs. Paediatric and geriatric are more vulnerable to drugs related ADRs due to age related pharmacokinetic and pharmacodynamics parameters and also due to poly pharmacy and irrational use of drugs. ${ }^{8}$

\section{METHODS}

The present study has been carried out after obtaining permission from institutional ethical committee.

The material is the pretested questionnaire on knowledge, attitude, practice on ADRs and PvPi. The design of the study is the cross sectional study. ${ }^{9}$ The setting and duration of the study is among the student of the $2^{\text {nd }}$ professional MBBS students during the month of December 2015. The sample size is intended to cover all the students of $2^{\text {nd }}$ professional MBBS students. The exclusion criteria are refusal to give consent, nonavailable or fail to return questionnaire in spite of the reminder and unable to attempt the questions. Percentage, proportions and means are used for descriptive statistics while the associations are calculated by using corresponding tests for the associations. ${ }^{10}$

\section{RESULTS}

There are 16 questions on knowledge but only 8 questions on ADRs, OTC/Self-medication, marketing, surveillance; CME and ADRs on selected population are selected for statistical analysis. The knowledge of the students on $\mathrm{PvPi}$ in connection with OTC/Self-medication (52\%), need of surveillance on marketing $(74 \%)$ but the surveillance on marketing may be low (60\%), need of CME on ADRs along with PvPi (64\%) as the ADRs on elderly (57\%), children (58\%), Pregnancy (64\%). Similarly out of 24 questions on Attitude, only 7 questions on reporting, MNC, DC (I), Disability, and Compensation are selected for statistical analysis. The attitude of students towards the compensation on account of ADRs is only $15 \%$ as it is the responsibility of the prescribers in terms of rational use of drugs, OTC/selfmedication, and surveillance. PvPi is very much useful to the MNC/DC (I) in view of the safety of the drugs during the marketing and post marketing surveillance and controlling the standards of the drugs under Drug and Cosmetics Acts. PvPi will be useful in prevention of disability due to ADRs as up to $70 \%$. Likewise there are 15 questions on Practice. Only 10 questions on definition of Pharmacovigilance, ADR, OTC/ self-medication, Marketing, usefulness to the MNC, DC (I),rational use of drugs and prevention from disability is picked up for statistical analysis. Result of the data analysis is shown on (Table1).

\section{Table 1: Percentage distribution of different parameters.}

\begin{tabular}{|lll|}
\hline Parameters & Yes & No \\
\hline Knowing definition of PvPi & 95.7 & 4.3 \\
\hline Definition of ADR/ADE & 88.8 & 11.2 \\
\hline Usefulness of ADR/ADE & 95.7 & 4.3 \\
\hline Usefulness of post Mkt & 90.1 & 9.9 \\
\hline Compensation of ADR/ADE & 27.5 & 72.5 \\
\hline Initiative and willingness & 88.8 & 11.2 \\
\hline Compensation & 27.5 & 72.5 \\
\hline
\end{tabular}

A Bar graph showing the different distribution of percentage of the students having the knowledge and attitude on ADRs, Rational use of drugs, post marketing surveillance, PvPi, Initiative and willingness of reporting, $\mathrm{CME}$ and Compensation is presented for reference. 


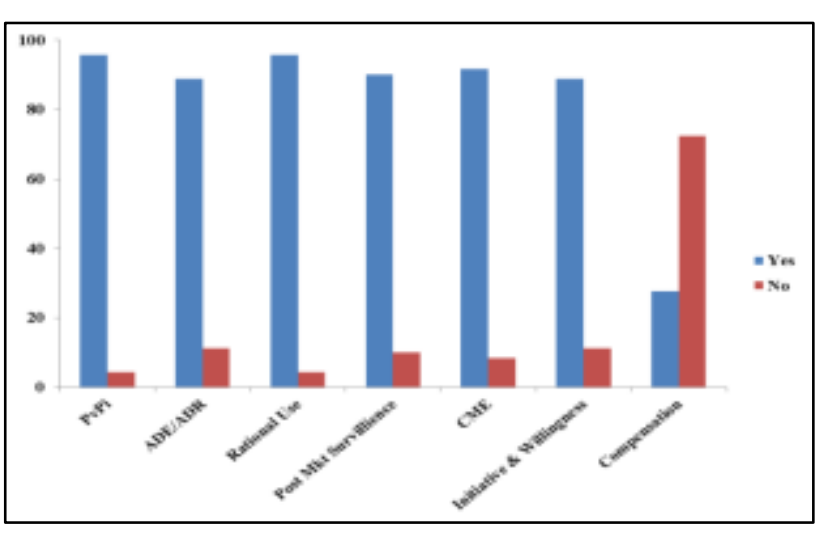

Figure 1: A bar graph.

Table 2: Correlation of attitude and practice on different parameters.

\begin{tabular}{|lcc|}
\hline Parameters & Value & Significance level \\
\hline $\begin{array}{l}\text { Usefulness of PvPi to } \\
\text { MNC }\end{array}$ & 0.87 & 0.01 \\
\hline $\begin{array}{l}\text { Usefulness of PvPi to } \\
\text { DCI }\end{array}$ & 0.23 & 0.05 \\
\hline
\end{tabular}

Table 3: Correlation of knowledge and practice on different parameters.

\begin{tabular}{|lll|}
\hline Parameters & Value & Significance level \\
\hline Common with OTC & 0.55 & 0.01 \\
\hline Marketing of drugs & 0.46 & 0.01 \\
\hline
\end{tabular}

\section{DISCUSSION}

The knowledge and attitude on ADRs, the willingness of the prescribers in prescription writing, reporting and notification of the ADRs is not to the marks. ADRs on OTC/self-medication (54\%), disability (73\%) may be prevented by practicing the rational use of drugs, caring of prescription during buying/marketing. Statistical study on the correlation between knowledge and practice; Attitude and Practice is shown in (Table 2 and 3). While examining the usefulness of the PvPi to the MNCs and the DC (I), the significance level is value 0.87 (p-0.01), and value 0.23 (p -0.05), the knowledge of students towards the practice on OTC and marketing is value $0.55(\mathrm{p}-0.01)$ and value 0.46 (p-0.01) shown as (Table 2 and 3). These indicate knowledge, attitude and practice of the ADRs/ $\mathrm{PvPi}$ and also willingness/initiative of the health care providers towards the implementation of the PvPi and the chances of the compensation may be as low as below $27.5 \%$.
Most of the ADRs may be avoided if there is good communication between the patients and health care providers. ADRs can be certainly minimised by considering the age related physiological changes and also pharmacokinetic/pharmacodynamics factors before prescribing the drugs.

\section{CONCLUSION}

From the present study, there are ample room for improvement and lot of works has to be done at the grass root level. Teaching on ADRs will be useful in educating the medical students, doctors and the officials working in controlling and regulating the sale and supply of drug. Therefore, as far as possible pharmacovigilance programme should be mandatory in each and every state health care institution.

\section{Funding: No funding sources}

Conflict of interest: None declared

Ethical approval: The study was approved by the Institutional Ethics Committee

\section{REFERENCES}

1. Tripathi KD. Adverse drug effects: - Essential of medical pharmacology, $7^{\text {th }}$ edition; 2013:82-83.

2. Bennett PN, Brown MJ. Unwanted effects and adverse drug reactions; Clinical pharmacology. $9^{\text {th }}$ edtion; 2003:135-150.

3. Hardman JG, Limbird LE, Gilman AG. Principles of therapeutic, the pharmacological basis of therapeutic. $10^{\text {th }}$ Edition; 2001:45-66.

4. Laurence DR, Bacharach AL. Toxicity tests; evaluation of drug activities; pharmacometrics. 2011;1:135-66.

5. Gaitonde BB, Telang BV. Drug toxicity and drug interactions. Basic and clinical pharmacology and therapeutics; 2010:42-50.

6. Gosh MN. Statistical analysis; fundamentals of experimental pharmacology $3^{\text {rd }}$ edi.; 2005:218-229.

7. National pharmacovigilance protocol, MOHFW, GOI.

8. MW Ahmed, Vardhamane SH, Dass P, Ahmed R, Gulabani M. Knowledge, attitude and practice of pharmacovigilance in baseveshwar teaching and general hospital: Journal of evolution of medicine and dental science. 2014;13(24):6698-707.

9. Park K. Text book of preventive and social medicine, $23^{\text {rd }}$ edition; 2015:72-79.

Cite this article as: Valte V, Laishram B, Singh PM, Raleng I. Pharmacovigilance studies on the basic knowledge, practice and attitude among the second year MBBS students of Jawaharlal Nehru institute of medical sciences, Porompat, Manipur, India. Int J Basic Clin Pharmacol 2016;5:820-2. 